\title{
Temperature Distribution in Core and Winding of a Transformer and Its Effect on Performance
}

\author{
Mahmmod Aziz Muhammed $^{1}$ and Abdul Ridha Ghedayer ${ }^{2}$ \\ Technical Institute/ Imara
}

\begin{abstract}
In order to reach the maximum power from a transformer and avoid thermal accidents, it is essential to carefully study its thermal behavior. This research is to apply some computational and analytical approaches in order to obtain a temperature distribution within the core and winding of a transformer. Hence its thermal behavior can be analytically and practically predicted. The aim is to optimize transformer operation under various load conditions during the extreme weather of summer season in the southern part of Iraq. The numerical scheme applied which is based on finite difference method has shown to be in good agreement with the empirical data measured on the external finned body of transformer.
\end{abstract}

Key Words: temperature distribution, finite difference method, distribution transformer, heat transfer, peak load condition.

\section{توزيع درجة الحرارة في القلب الحديدي والملقات لمحولة كهربائية وتأثيره على الألى الاد|و}

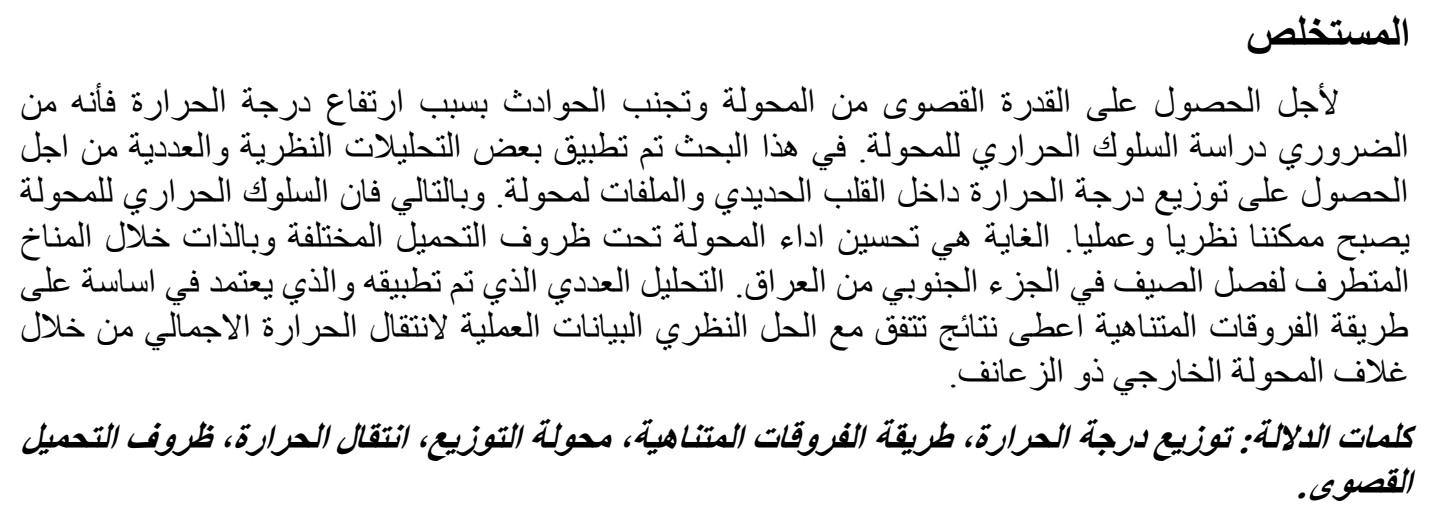

\section{Introduction}

A transformer as an electrical device has a complicated design with specific interconnected elements and parts, and various physical phenomena that describe the real system behavior. In many cases, demands for its proper operation force reconsideration of conventional design methods to include complexity of non-linear materials, composite geometry structures, operation at transient and steady-state 
conditions. The prediction and determination of temperature distribution in the metal parts of transformers is however a very important step in the process of designing equipment. Thermal design of a transformer is carried out using an empirical method which is based on a calculation of the top oil and top winding temperatures. This method involves extrapolating thermal data with empirical equations to calculate the top oil and top winding temperatures. Nearly all the power and distribution transformer manufacturers use this method as well as thermal tests known as heat run tests. The idea behind this research is that transformer faults always cause internal damage. The basic criterion that limits transformer loading and its lifetime is partially determined by the ability of the transformer to dissipate internally generated heat into the surrounding area. Therefore, it is of great importance to predict the thermal behavior of a transformer under normal load. The thermal effect on transformer performance is studied using numerical analysis in order to predict the temperature distribution within the transformer; namely windings and iron core. Hence the heat transfer through the transformer to the surroundings can be calculated.

\section{Literature Review}

Computational methods and engineering models proposed for transformer analysis and the accurate prediction of their characteristics can be categorized into four main groups:

1. Numerical techniques that consist some of the most widely used tools for transformer simulation. Among the proposed techniques of this group, the Finite Difference Method (FDM) represents the easiest one.

2. Stochastic methods including Artificial Intelligence (AI) techniques, such as Genetic Algorithms (GA), which have seen increased usage in the transformer design area over the last few years.

3. Versions of the transformer equivalent circuit. Its use is still common in the manufacturing industry, due to its simplicity and its ability to provide reliable results, especially in cases of standardized geometries.

4. Experimental methods, combining data provided by measurements with analytical or other methods, in order to provide efficient models for the accurate representation of certain transformer characteristics.

Numerical methods although more precise in the calculation of oil and winding temperatures of a transformer, they have been considered as a complementary calculation to the empirical or thermal test [1 and 2]. Pierce developed a network model which produces a set of algebraic equations based on a heat balance performed in every node considered in the region of interest. The thermal convective coefficients were considered from standard correlations found in the literature. Later this set of finite difference equations were solved for the temperatures using standard algorithms for solution of the resulting matrices. Other researchers used a less traditional approach by means of the heat conduction equation and its solution through advanced mathematical techniques [3]. 
El-Wakil et al [4], proposed a numerical model which was solved using the FLUENT program for a $100 / 22.5 \mathrm{kV}$ power transformers. He analyzed some interesting geometries composed of top and bottom barriers within the low and high voltage windings which yield different oil velocities. Olsson [5] analyzed a disc type winding of a power transformer to determine the influence of oil streams coming out from the horizontal oil ducts which were believed to modify the oil velocity. The analysis is performed in a two dimensional model of a disc composed of eighth conductors. The momentum and energy equations are solved by the FLUENT program. Haritha [6] proposed a numerical model and solved it with NISA program for a $500 \mathrm{KVA}$ onephase transformer. In this research, a numerical scheme based on the work in [7] is proposed for the iron core and windings of a transformer. Two coordinate systems; Cartesian for the transformer iron core and cylindrical coordinates for the windings are considered. The thermal resistance concept is then applied at the boundaries because it offers more flexibility than the conventional finite difference scheme even though the discretization of governing equations is mainly based on finite difference method. This scheme sounds new and has never been applied by other researchers. Moreover and different to the above mentioned works, the temperature distribution is plotted in two dimensions because the temperature along the height of coil is assumed not constant.

\section{Theoretical Model}

Distribution transformers are used to convert electrical energy from a high to a low voltage. In Iraq these levels of voltage vary from 11 to $33 \mathrm{kV}$ in the higher level to $0.416 \mathrm{kV}$ in the lower level. The internal structure of a transformer is complex and it does not fit any known geometry. A pictorial description of $400 \mathrm{kVA}$ distribution transformer is shown in Fig. 1 The internal geometry is composed of rectangular core and co-axial cylinders for high and low voltage windings. Besides, the windings are made of layers of copper or aluminum and among them a layer of insulating NOMEX paper to electrically isolate every turn of copper or aluminum layer. 


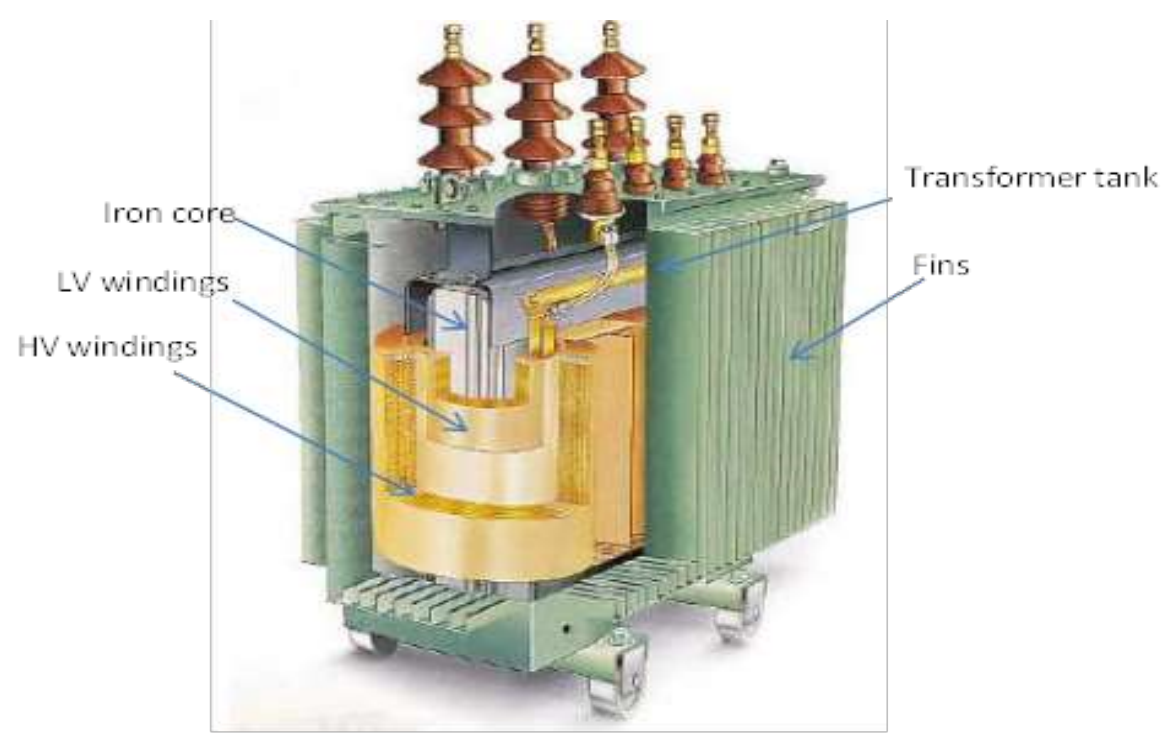

Fig. (1) Internal geometry of a $400 \mathrm{kVA}$ transformer

The two dimensional model proposed for this transformer is a rectangular slice which is composed of the corresponding section of core, high and low voltage windings, see Fig. 2. The HV windings are located in the surrounding space of LV windings which is filled by oil as an electrical insulation and heat dissipation media. The numerical values of the parameters shown in Fig. 2 are reliant on the size of transformer, i.e. the power capacity of transformer.

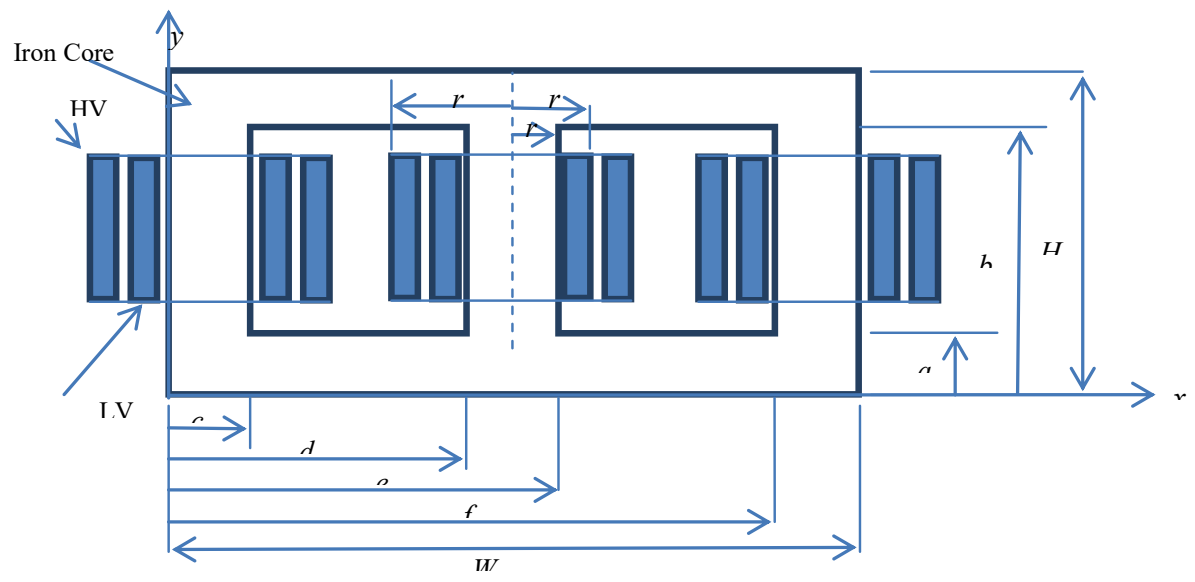

Fig. (2) Two Dimensional Model with Three Windings and iron core Of The Transformer shown in Figure (1) 
The identification of oil-immersed transformers according to the cooling method consist the following information:

- Type of internal cooling medium in contact with the windings

$\circ$ Identification of the circulation mechanism for internal cooling medium and external cooling medium

○ Identification of the circulation mechanism for external cooling medium.

For example, if the internal cooling medium is mineral oil, which is circulated with natural flow, and the external cooling medium is air, which is circulated with natural convection, then this cooling method is coded as ONAN (Oil Natural Air Natural). A four cooling methods exist: ONAF (Oil Natural Air Forced), OFAN (Oil Forced Air Natural), OFAF (Oil Forced Air Forced) and OFWF (Oil Forced Water Forced).

\subsection{Heat Equation}

The following equation is applied for steady state heat conduction equation within iron core of the transformer [8]

$$
\frac{\partial^{2} T}{\partial x^{2}}+\frac{\partial^{2} T}{\partial y^{2}}=0
$$

The boundary conditions related to this equation are:

$$
\begin{array}{ll}
-k_{c} \frac{d T}{d x}=h\left(T_{\text {core }}-T_{\text {oil }}\right) & \text { At } x=0 \text { and } x=W, a>y>b \\
-k_{c} \frac{d T}{d x}=\overline{\bar{q}}_{\text {windings }} & \text { At } x=0, x=c, x=d, x=e, x=f, x=W ; a \leq y \leq b \\
-k_{c} \frac{d T}{d y}=h\left(T_{\text {core }}-T_{\text {oil }}\right) & \text { At } y=0 \text { and } y=H \\
-k_{c} \frac{d T}{d y}=\overline{\bar{q}}_{\text {windings }} & \text { At } y=a \text { and } y=b, c \leq x \leq d \text { and } e \leq x \leq f
\end{array}
$$

Where $\overline{\bar{q}}_{\text {windings }}$ is the constant heat flux of windings, $k_{c}$ is the thermal conductivity of iron core. The constants $a, b, c, d$, e and $f$ are shown in Fig. 2.

For transformer's windings, the equation applied can be expressed as follows:

$$
\frac{\partial^{2} T}{\partial r^{2}}+\frac{1}{r} \frac{\partial T}{\partial r}+\frac{\partial^{2} T}{\partial z^{2}}+\frac{q_{g}}{k_{w}}=0
$$

With boundary conditions:

$$
\begin{array}{ll}
-k_{w} \frac{d T}{d r}=\overline{\bar{q}}_{\text {core }} & \text { at } r=r_{0} \\
-k_{w} \frac{d T}{d r}=\overline{\bar{q}}_{\text {windings }} & \text { at } r=r_{1} \\
-k_{w} \frac{d T}{d r}=h\left(T_{\text {windings }}-T_{\text {oil }}\right) & \text { at } r=r_{2}
\end{array}
$$

Where $\overline{\bar{q}}_{\text {core }}$ is the constant heat flux of iron core, $k_{w}$ is the thermal conductivity of windings, $r_{0}, r_{1}, r_{2}$ are constants shown in Fig. 2. 
Boundaries for this analysis are composed of the internal boundaries conformed by the $\mathrm{HV} / \mathrm{LV}$ windings and core, and external boundaries which are composed of the tank walls and radiator fins. This is shown in Fig. 3.

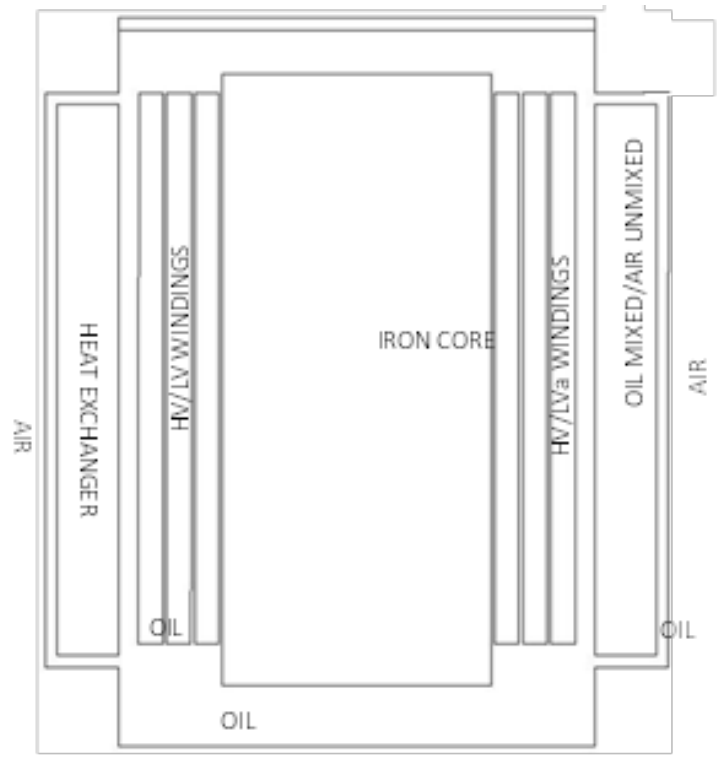

Fig. (3) Internal and external boundaries in a transformer

The volumetric heat source function $\left(\dot{q}_{g}\right)$ has been modified to take care of variation of electrical resistance of copper with temperature. The heat source term $\dot{q}_{g}$ can be of the form:

$$
\dot{q_{g}}=\dot{q}_{0}\left[1+\alpha_{e}\left(T-T_{r e f}\right)\right]
$$

Where $\alpha_{e}$ is the temperature coefficient of electrical resistance of copper wire, $T_{r e f}$ is the reference temperature and $\dot{q}_{0}$ is the initial value of heat generation. With this representation, the function $\dot{q}_{g}$ becomes temperature dependent (i.e. distributed heat source).

\subsection{Heat Convection Equation}

For the heat convection at the internal boundaries the following equation is applied for oil:

$$
\rho\left(V_{x} \frac{\partial\left(c_{p} \cdot T\right)}{\partial x}+V_{y} \frac{\partial\left(C_{p} \cdot T\right)}{\partial y}\right)=\frac{\partial}{\partial x}\left(k \cdot \frac{\partial T}{\partial x)}\right)+\frac{\partial}{\partial y}\left(k \cdot \frac{\partial T}{\partial y)}\right)
$$

Where $T$ is temperature, $V_{x}$ is velocity in $x$ direction, $V_{y}$ is velocity in $y$ direction, $k$ is thermal conductivity, $\rho$ is density and $C_{p}$ is specific heat of the transformer's oil.

In equation (4) velocity is unknown, and then we must solve Navier-Stokes equations. Navier-Stokes equations in two dimensions for Newtonian incompressible fluid are: 
Continuity Equation:

$$
\frac{\partial V_{x}}{\partial x}+\frac{\partial V_{y}}{\partial y}=0
$$

Momentum Equations:

$$
\begin{gathered}
\rho\left(V_{x} \frac{\partial V_{x}}{\partial x}+V_{y} \frac{\partial V_{y}}{\partial y}\right)=-\frac{\partial P}{\partial x}+\left(\frac{\partial}{\partial x}\left(2 \cdot \mu \cdot \frac{\partial V_{x}}{\partial x}\right)+\frac{\partial}{\partial y}\left(\mu \cdot \frac{\partial V_{x}}{\partial y}+\mu \cdot \frac{\partial V_{y}}{\partial x}\right)\right) \\
\rho\left(V_{x} \frac{\partial V_{x}}{\partial x}+V_{y} \frac{\partial V_{y}}{\partial y}\right)=-\frac{\partial P}{\partial y}+\left(\frac{\partial}{\partial x}\left(\mu \cdot \frac{\partial V_{x}}{\partial y}+\mu \cdot \frac{\partial V_{y}}{\partial x}\right)+\frac{\partial}{\partial y}\left(2 \cdot \mu \cdot \frac{\partial V_{y}}{\partial y}\right)\right) \\
+\rho_{0} \cdot g \cdot \beta \cdot\left(T-T_{r e f}\right)
\end{gathered}
$$

Where $P$ is pressure and $g$ is gravitational acceleration.

\subsection{Heat Exchanger}

At the external boundaries, the following equation is applied [11]

$$
q_{\text {actual }}=\epsilon \cdot C_{\min }\left(T_{\text {oil }}-T_{\infty}\right)
$$

Where:

If $C_{\max }$ is mixed, $C_{\min }$ unmixed

Then $\epsilon=\left(\frac{1}{C}\right)\left\{1-\exp \left[-C\left(1-e^{-N}\right)\right]\right\}$

If $C_{\max }$ is unmixed, $C_{\min }$ mixed

Then $\epsilon=1-\exp \left\{-\left(\frac{1}{C}\right)[1-\exp (-N C)]\right\}$

Note: $\quad N=N T U=\frac{U A}{C_{\min }} \quad C=\frac{C_{\min }}{C_{\max }}$

For the calculation of $U$ we need the convective heat transfer coefficient $h$ which can be calculated using the following equations:

$$
\begin{aligned}
& \bar{N}_{u_{L}}^{\frac{1}{4}}\left(\overline{N_{u_{L}}}-0.68\right)=\frac{0.67\left(G r_{L}^{*} P r\right)^{1 / 4}}{\left[1+(0.492 / P r)^{\frac{9}{16}}\right]^{4 / 9}} \\
& G r_{L}^{*}=G r_{x} N u_{x}=\frac{g \beta^{*} q_{w} x^{4}}{k v^{2}}, \quad \beta^{*}=\frac{1}{T}
\end{aligned}
$$

The later equations are used to compare the heat dissipated by the core and windings to the heat loss through the external finned body.

\section{Numerical Model}

In the previous section, it was mentioned that the total heat generated in the HV/LV windings and core is dissipated in the oil and then to the surrounding air via external heat exchanger. Values of temperature distribution within the core are made known 
using the finite Difference method scheme which can be written for equation (1) as follows [8]

$$
\frac{\left(T_{m+1, n}-2 T_{m, n}+T_{m-1, n}\right)}{\Delta x^{2}}+\frac{\left(T_{m, n+1}-2 T_{m, n}+T_{m, n-1}\right)}{\Delta y^{2}}+\tau(\Delta x)^{2}+\tau(\Delta y)^{2}=0
$$

If $\Delta x=\Delta y$ the above equation becomes:

$$
T_{m+1, n}+T_{m-1, n}+T_{m, n+1}+T_{m, n-1}-4 T_{m, n}=0
$$

The truncation error associated with this representation has the order of quadratic, i.e. $\tau(\Delta x)^{2}$ and $\tau(\Delta y)^{2}$ because both derivate operators in $x$ and $y$ are discretized using central difference equation. For the subscript $m$ and $n$ see figure 4 below with a notice $\Delta x=\Delta y$

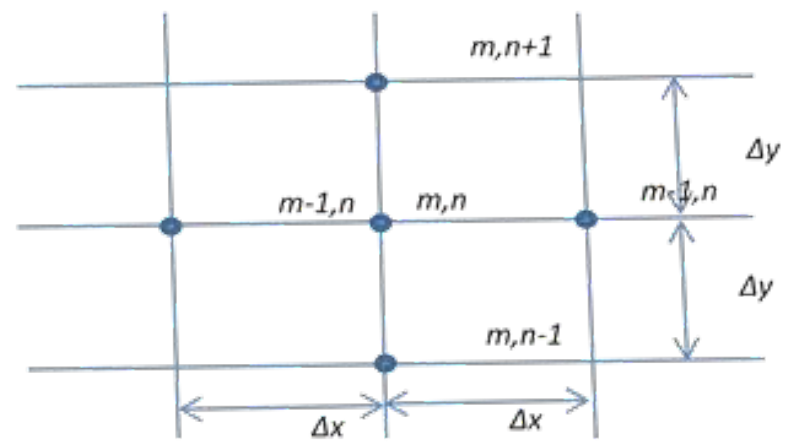

Fig. (4) A schematic drawing illustrating nomenclature used in two dimensional numerical analysis of equation (9)

The discretization of equation (2) which governs the temperature distribution in LV and $\mathrm{HV}$ windings can be expressed as follows:

$$
\begin{gathered}
\left(\frac{T_{m+1, n}-2 T_{m, n}+T_{m-1, n}}{(\Delta r)^{2}}\right)+\frac{1}{(N-1) \Delta r}\left(\frac{T_{m+1, n}-T_{m-1, n}}{2 \Delta r}\right)+\left(\frac{T_{m, n+1}-2 T_{m, n}+T_{m, n-1}}{(\Delta z)^{2}}\right)+\frac{\dot{q}_{g}}{k_{w}}+ \\
\square(\Delta r)^{2}+\square(\Delta z)^{2}=0
\end{gathered}
$$

The discretization of first derivate in $r$ direction is expressed in central difference equation in order to keep the error at the quadratic power. The above equation can be simplified further as follows:

$$
\frac{(2 N-1)}{(2 N-2)} T_{m+1, n}+\frac{(2 N-3)}{(2 N-2)} T_{m-1, n}+(2 N-2)\left[\left\{T_{m, n-1}+T_{m, n+1}-4 T_{m, n}\right\}+\frac{\dot{q}_{g}(\Delta r)^{2}}{k_{w}}\right]=0
$$

Where $N$ is the number of grids which is equal in both $r$ and $z$ directions, i.e. $\Delta r=\Delta z$. it is also should be noticed that $m$ and $n$ stand for the discretization in $r$ and $z$ respectively. The truncation error for this equation is of quadratic power both in $r$ and $z$ directions. 
The notation $\square$ expresses the rate at which the truncation error goes to zero. Usually we are only interested in the order of truncation error. For code validation, however, we need to work with the magnitude of the truncation error. Let $T E$ denotes the true magnitude of the truncation error for a given $\Delta x$ and $\Delta y$ (or alternatively $\Delta r$ and $\Delta z$ ). As $\Delta x \rightarrow 0$ and $\Delta y \rightarrow 0$, the true magnitude of the truncation error is

$$
T E=K_{x}(\Delta x)^{2}+K_{x}(\Delta y)^{2}
$$

Where $K_{x}$ and $K_{y}$ are constants that depend on the accuracy of numerical approximations and the problem being solved. To make $T E$ arbitrarily small, both $\Delta x$ and $\Delta y$ must approach zero.

To calculate the percentage of error between the measured and calculated data, the following equation is applied:

$$
\text { error } \%=\left|\frac{T_{\text {measured }}-T_{\text {computed }}}{T_{\text {measured }}}\right|
$$

At the boundaries and for both systems the concept of resistance between two adjacent nodes is applied. The general equation for this concept is [11]

$$
\dot{q}+\sum_{j} \frac{T_{j}-T_{i}}{R_{i j}}=0
$$

Where $\dot{q}$ is the time rate of heat conduction and the subscript $i$ is designated for the node of interest while $j$ refers to the adjoining node. $R_{i j}$ is the resistance between two adjoining nodes and it expressed in plus and minus signs where plus means the positive direction of coordinate and minus is the negative direction. Table 1 lists the formula for each resistance according to the type of boundary conditions.

Table (1) resistance formulas for convection boundary conditions

\begin{tabular}{|r|r|c|c|c|c|}
\hline System & Boundary conditions & $R_{m+}$ & $R_{m-}$ & $R_{n+}$ & $R_{n-}$ \\
\hline Iron Core & Convection boundary & $\frac{1}{h \Delta x}$ & $\frac{1}{k}$ & $\frac{2}{k}$ & $\frac{2}{k}$ \\
\cline { 2 - 6 } & $\begin{array}{r}\text { Exterior corner } \\
\text { convection }\end{array}$ & $\frac{2}{h \Delta x}$ & $\frac{2}{k}$ & $\frac{2}{h \Delta y}$ & $\frac{2}{k}$ \\
\cline { 2 - 6 } & $\begin{array}{r}\text { Interior corner } \\
\text { convection }\end{array}$ & $\frac{2}{k}$ & $\frac{1}{k}$ & $\frac{1}{k}$ & $\frac{2}{k}$ \\
\hline $\begin{array}{r}\mathrm{HV} / \mathrm{LV} \\
\text { windings }\end{array}$ & Convection boundary & $R_{m+}$ & $R_{m-}$ & $R_{k+}$ & $R_{k-}$ \\
\cline { 2 - 6 } & & $\frac{r_{m} \Delta \emptyset}{\Delta r \Delta z k}$ & $\frac{r_{m} \Delta \emptyset}{\Delta r \Delta z k}$ & $\frac{\Delta z}{r_{m} \Delta \emptyset \Delta z k}$ & $\frac{\Delta z}{r_{m} \Delta \emptyset \Delta z k}$ \\
\hline
\end{tabular}




\subsection{Assumptions}

The following assumptions are applied to the analysis shown above in order to obtain the temperature distribution in iron core and windings:

- For both systems the grids are equal and for windings $\Delta \emptyset$ is considered constant $(\Delta \emptyset=2 \pi)$. This assumption comes from the symmetrical design of transformer which yields constant distribution of temperature along the winding direction.

- The oil inside transformer undergoes natural convection which pushes the hot oil above and replaces it with colder oil at the bottom. Hence, temperature distribution in $z$ direction is considered not constant.

- The region inside the LV/HV windings and core is so stagnant that only heat conduction is considered. i.e. the only term considered in heat conduction is the right side of equation (4).

- The space between two adjacent windings is so narrow that the oil filled this space can be perfectly assumed as a stagnant viscos fluid hence, the conduction heat transfer is dominant. Therefore, the boundary conditions for the middle windings (normally phase $\mathrm{S}$ ) is expressed as follows:

$$
-k_{w} \frac{d T}{d r}=\overline{\bar{q}}_{\text {windings }} \quad \text { at } r=r_{2}
$$

- Initially, the whole system is considered at the atmospheric temperature (outdoor summer temperature is $55^{\circ} \mathrm{C}$ ) in order to simulate the initial condition of the transformer and to observe the evolution of the system. However, since the evolution time is not too long in comparison with the time the transformer reaches its steady state operation; the final condition was considered as a steady state condition.

\section{Results And Discussion}

Before proceeding with results and discussion, the technical specifications of the transformer under test are listed in table (2)

Table (2) Technical Data of Transformer

\begin{tabular}{|c|c|c|c|}
\hline No. & Description & Unit & Requirements \\
\hline 1 & Type & $\begin{array}{c}\text { 3-ph oil immersed } \\
\text { without conservator }\end{array}$ \\
\hline 2 & $\begin{array}{c}\text { Continuous Max Rating } \\
\text { (CMR) }\end{array}$ & $\mathrm{kVA}$ & 400 \\
\hline 3 & Rated Frequency & $\mathrm{Hz}$ & 50 \\
\hline 4 & Cooling Method & & ONAN \\
\hline 5 & High Voltage (HV) & $\mathrm{V}$ & 11000 \\
\hline
\end{tabular}




\begin{tabular}{|c|c|c|c|}
\hline 6 & Low Voltage (LV) & $\mathrm{V}$ & 400 \\
\hline 7 & HV Windings & & Delta \\
\hline 8 & LV Windings & & Star \\
\hline 9 & off Load Losses & W & 610 \\
\hline 10 & On Load Losses & W & 3850 \\
\hline 11 & Top Oil Temperature & ${ }^{\circ} \mathrm{C}$ & 55 \\
\hline 12 & $\begin{array}{l}\text { Average Winding } \\
\text { Temperature }\end{array}$ & ${ }^{\circ} \mathrm{C}$ & 60 \\
\hline 13 & Hot Spot for windings & ${ }^{\circ} \mathrm{C}$ & 115 \\
\hline 14 & Installation & & Outdoor \\
\hline 15 & $\begin{array}{l}\text { Kinematic Viscosity of Oil at } \\
\qquad 40^{\circ} \mathrm{C}\end{array}$ & $\mathrm{mm} 2 / \mathrm{s}$ & 11 \\
\hline 16 & Density & $\mathrm{Kg} / \mathrm{m} 3$ & $\leq 895$ \\
\hline 17 & Breaking voltage of oil & $\mathrm{kV}$ & $>40$ \\
\hline 18 & Height of windings & $\mathrm{mm}$ & 300 \\
\hline 19 & Inner Radius of LV windings & $\mathrm{mm}$ & 100 \\
\hline 20 & $\begin{array}{l}\text { Outer Radius of LV } \\
\text { Windings }\end{array}$ & $\mathrm{mm}$ & 135 \\
\hline 21 & Inner Radius of HV Windings & $\mathrm{mm}$ & 140 \\
\hline 22 & $\begin{array}{l}\text { Outer Radius of HV } \\
\text { Windings }\end{array}$ & $\mathrm{mm}$ & 175 \\
\hline & Diameter of Iron Core & $\mathrm{mm}$ & 195 \\
\hline 18 & Length & $\mathrm{mm}$ & 1400 \\
\hline 19 & Width & $\mathrm{mm}$ & 850 \\
\hline 20 & Height & $\mathrm{mm}$ & 1050 \\
\hline 21 & Initial body temperature & ${ }^{\circ} \mathrm{C}$ & 63 \\
\hline 22 & Steady state body temperature & ${ }^{\circ} \mathrm{C}$ & 73 \\
\hline 23 & $\begin{array}{l}\text { Base temperature at finned } \\
\text { body }\end{array}$ & ${ }^{\circ} \mathrm{C}$ & 73 \\
\hline 24 & Ambient temperature & ${ }^{\circ} \mathrm{C}$ & 55 \\
\hline 25 & Temperature coefficient $\left(\alpha_{e}\right)$ & $\%$ & 0.393 per ${ }^{\circ} \mathrm{C}$ \\
\hline 26 & Initial heat generation $\left(q_{0}\right)$ & $\mathrm{W} / \mathrm{m} 3$ & 100 \\
\hline
\end{tabular}


The numerical scheme mentioned in the previous section was solved though programing codes using MATLAB software. The initial condition was set to a constant temperature throughout the transformer which is equal to the highest ambient temperature during summer season in Iraq (namely southern part of Iraq). This temperature was measured and it reached $55^{\circ} \mathrm{C}$ which represents the temperature stated as a maximum ambient temperature in the technical specifications of Ministry of Electricity - Iraq. However, the temperature of Transformer tank reached as high as $63^{\circ} \mathrm{C}$ (according the data collected by the researchers during August 2015). Hence, the constant initial temperature was set to $63^{\circ} \mathrm{C}$ which assumes that there was an interruption in power supply of three hours. At this initial condition there should be no efficient heat transfer between the transformer body and ambient temperature as they are in thermal equilibrium. As the electrical load grows up after power recovery, the temperature of transformer body increases until it reaches the effective temperature difference required by the heat exchanger at the finned body [11]. The transformer body temperature at steady state condition was measured at full load and it was between 82 and $85^{\circ} \mathrm{C}$. This temperature was assumed as a base temperature for the heat exchanger at the finned body. Temperature distribution within the middle windings (phase $\mathrm{S}$ ) and where the maximum temperature occurs according to winding height is shown in Fig. (5). The peak temperature occurred at a height of $165 \mathrm{~mm}$ from the winding lower edge and its value exceeded the design temperature of $115^{\circ} \mathrm{C}$, see table 4 above.

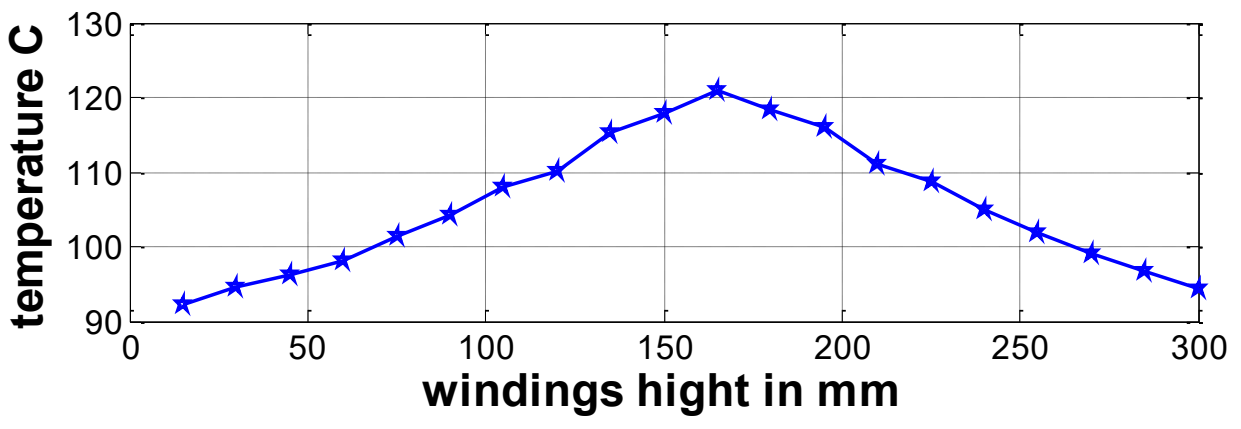

Fig. (5) Temperature Distribution along the height of S Phase Windings

Temperature distribution within the middle windings (phase $\mathrm{S}$ ) and where the maximum temperature occurs according to winding radius is shown in Figure (6). Peak temperature occurs near the outer boundary of LV windings and its value exceeded the design temperature of $115^{\circ} \mathrm{C}$, see table 4 above. 


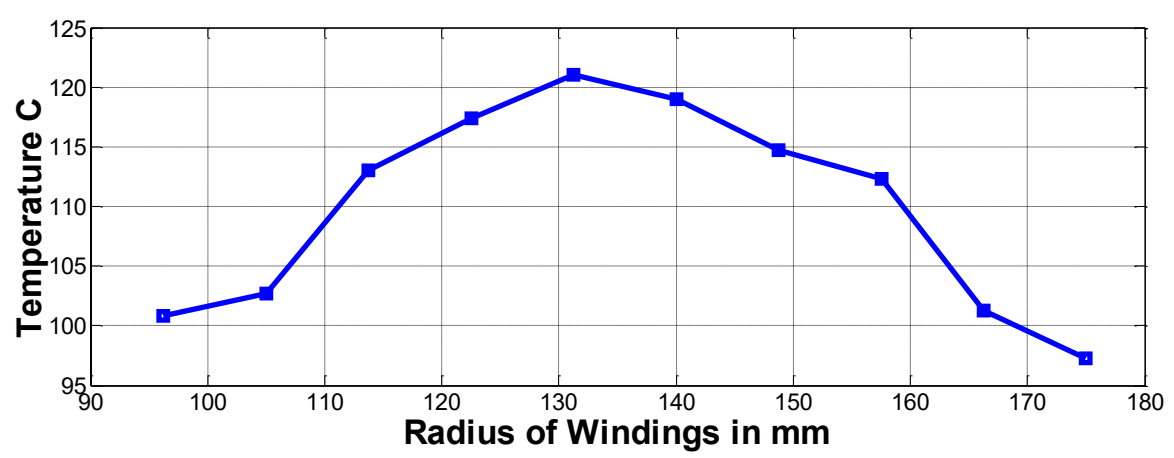

Fig. (6) Temperature Distribution along the radius of S Phase Windings

For better visualization, Figure (7) shows temperature distribution within S Phase windings with respect to radius and height of the windings. It should be noticed that LV windings is located inside HV windings, see Figure (2).

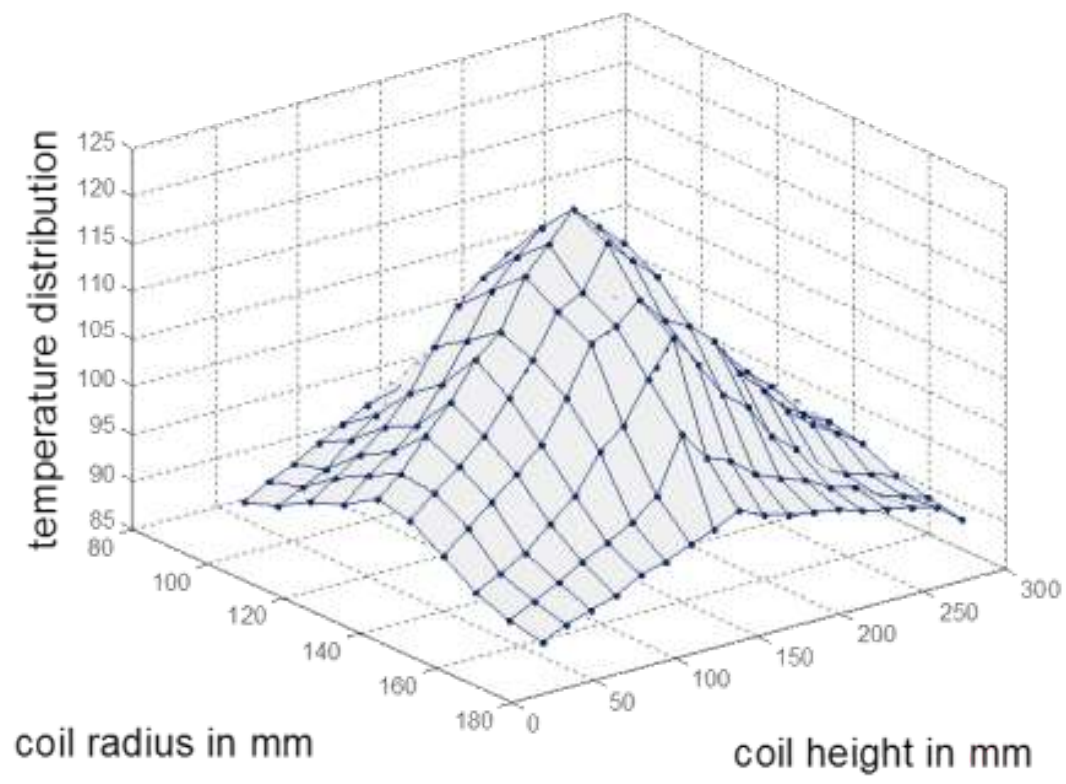

Fig. (7) Temperature Distribution within S Phase Windings with respect to radius and height of windings

The assumption of constant temperature distribution in $\emptyset$ direction converted the windings to two rectangles which interprets the parabolic distribution shown in Figure (8). For the iron core, the position where phase $\mathrm{S}$ is located undergoes the highest temperature. This fact is clearly shown in Figure (9) where one can compare the temperatures at each phase. 


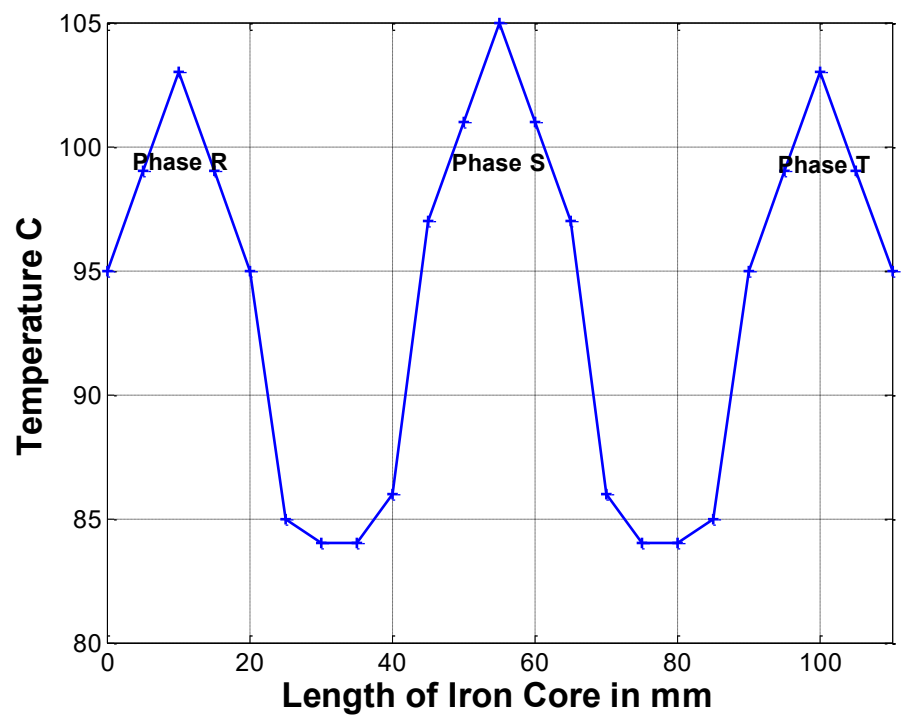

Fig. (8) Temperature Distribution along the iron core length

The same distribution is shown in Figure (9) but in both dimensions for clearer visualization.

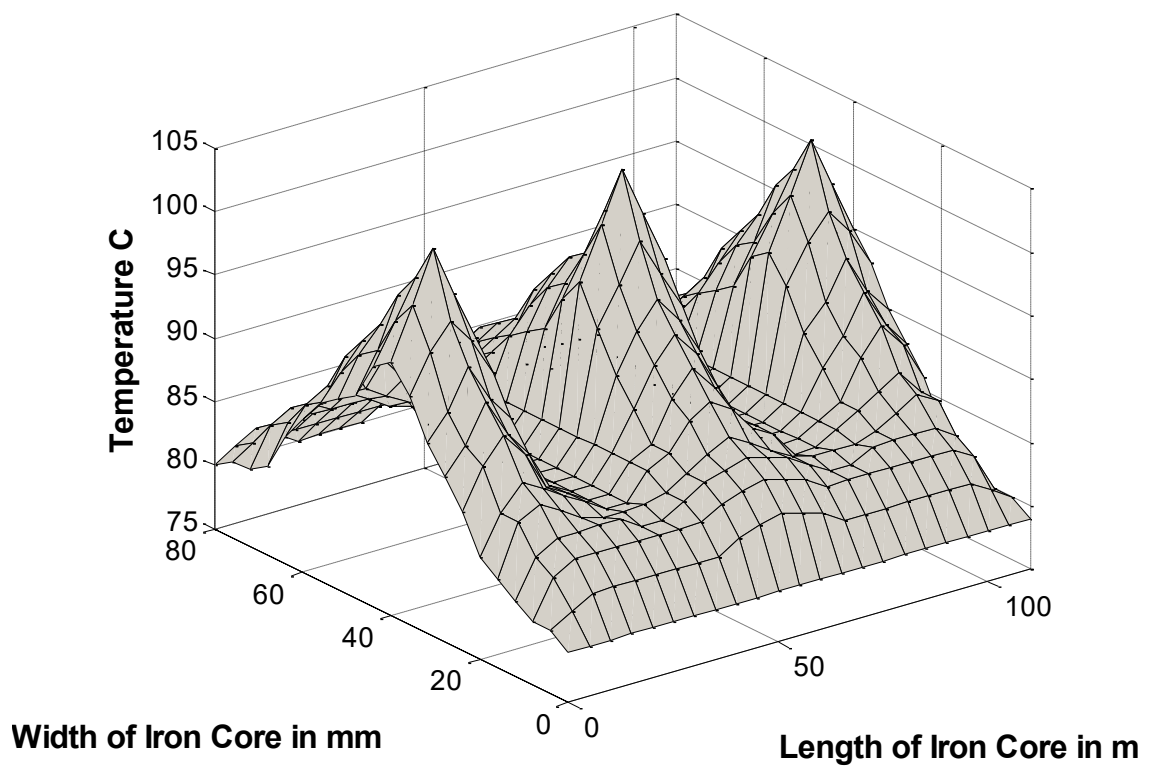

Fig.(9) Temperature Distribution in the iron core

Solving Navier - Stokes equation for the conduction term resulted in temperature gradient along the transformer height. This was in good agreement with the data taken onsite with assumption that the temperature of oil in the neighboring region of finned body is lower than the base temperature by $2-3{ }^{\circ} \mathrm{C}[11]$. 
The value of temperatures of oil obtained from solving Navier - Stokes equations (oil temperature calculated) and the temperature of transformer body (body temperature measured) along with oil temperatures according to body temperatures (oil temperature measured) are shown in Table (3).

Table (3) Numerical values of temperature as essential data for numerical scheme

\begin{tabular}{|c|c|c|c|c|}
\hline Height (m) & $\begin{array}{c}\text { Body Temp. } \\
\text { measured }\left({ }^{\circ} \mathbf{C}\right)\end{array}$ & $\begin{array}{c}\text { Oil Temp. } \\
\text { measured } \\
\left({ }^{\circ} \mathbf{C}\right)\end{array}$ & $\begin{array}{c}\text { Oil Temp. } \\
\text { calculated } \\
\left({ }^{\circ} \mathbf{C}\right)\end{array}$ & $\begin{array}{c}\text { ERROR } \\
\text { \% }\end{array}$ \\
\hline 0.1 & 75 & 73 & 70 & 5.55 \\
\hline 0.3 & 78 & 76 & 72 & 2.63 \\
\hline 0.5 & 80 & 78 & 76 & 5.19 \\
\hline 0.65 & 83 & 81 & 77 & 1.19 \\
\hline 0.75 & 85 & 83 & 84 & 2.29 \\
\hline 0.9 & 87 & 85 & 87 & 2.24 \\
\hline 1.0 & 89 & 87 & 89 & 5.55 \\
\hline
\end{tabular}

The numerical values of oil temperature are depicted in Fig. (10) as a comparison between the measured and calculated data.

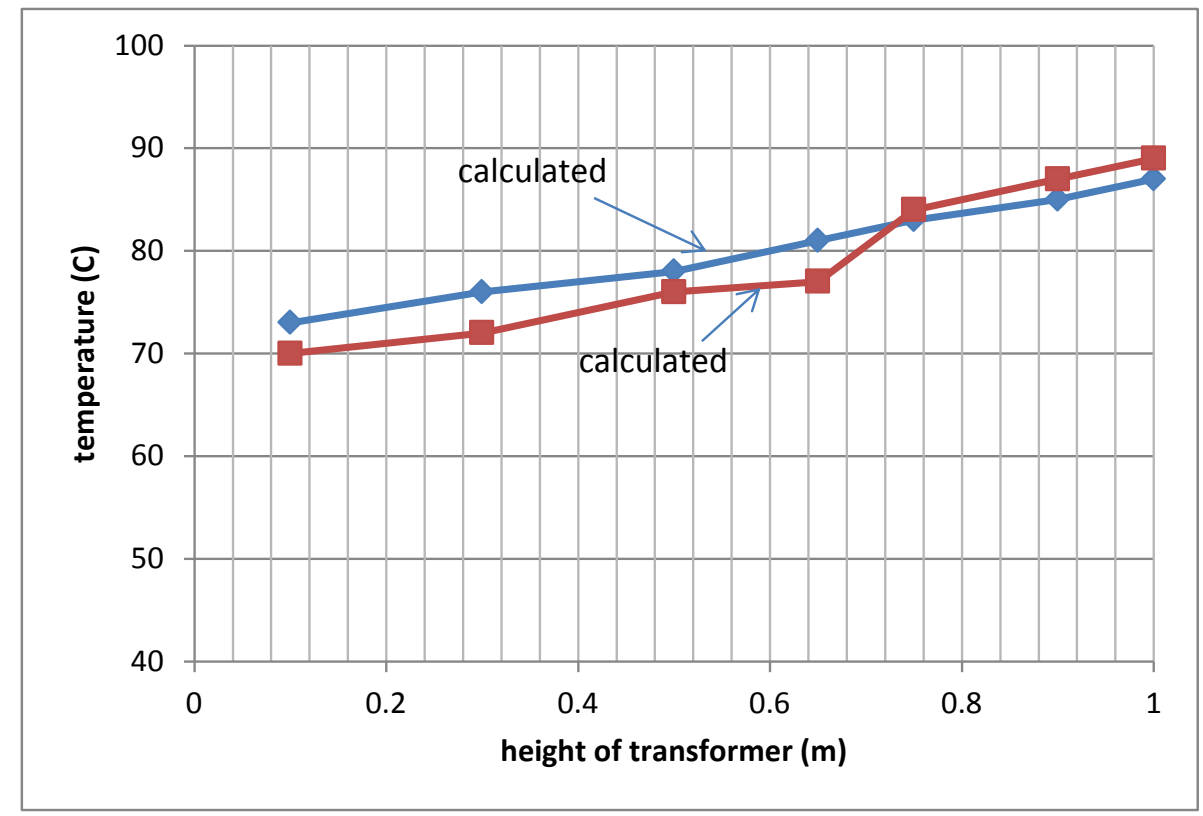

Fig. (10) A comparison between values of temperature measured and calculated 
The error associated with this data which is calculated using equation (12) is shown in Fig. 11. It is obviously noticed that the data is convergent near. The top of transformer (top finned tank of transformer) which can be interpreted as the fact that the top of transformer is normally filled with hot oil which its temperature is in equilibrium with body temperature.

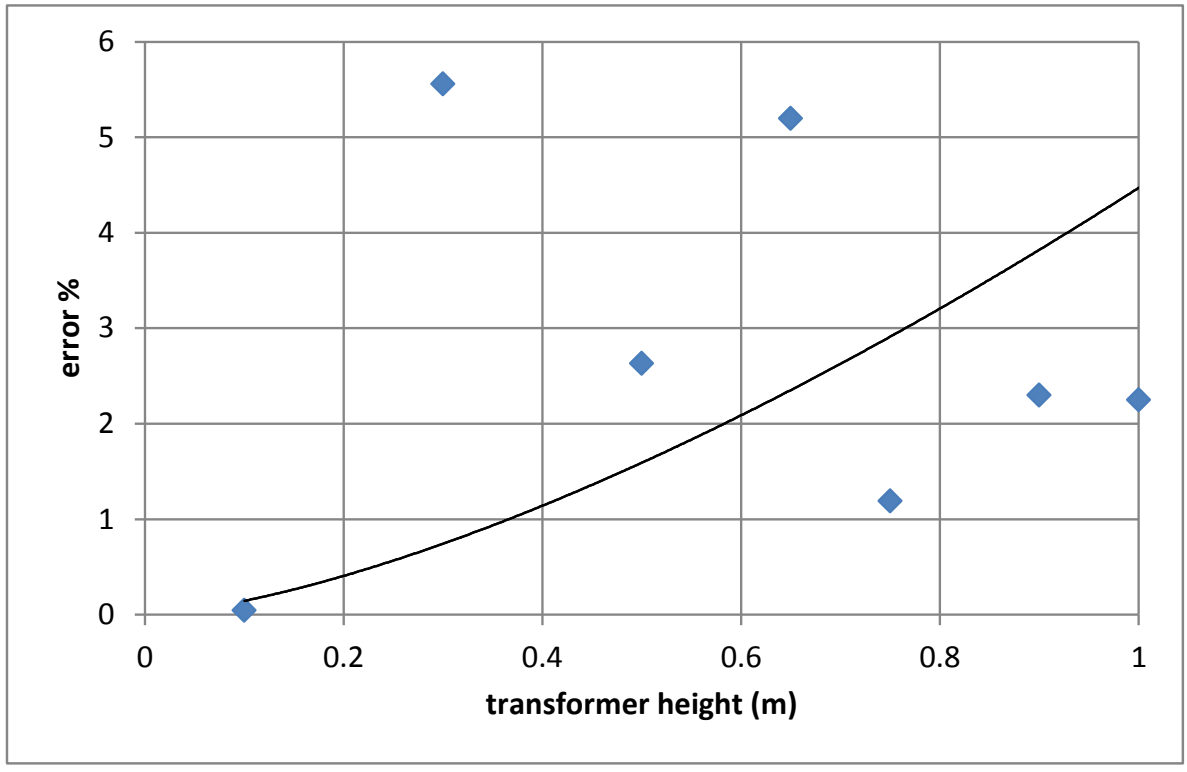

Fig. (11) The error of numerical scheme compared to data measured

According to the data shown in Figure (6) the maximum difference between the transformer's core and oil is 40 degrees. For the external heat transfer at the finned body, a maximum difference of 30 degrees is reached. However, the natural convective heat transfer coefficient for the oil is normally higher than that for moderate speed wind. The temperature of winding of phase $\mathrm{S}$ is taken as a surface temperature of internal components with a total area calculated from the data from table 3 that is equal to 2.593 $\mathrm{m}^{2}$ and convective heat transfer coefficient of oil $35 \mathrm{~W} / \mathrm{m}^{2} . \mathrm{K}$, the internal heat transfer during the day hours ranging from 6:00 AM to 4:00 PM can be calculated in order to be compared with the external heat which is calculated using equations $(6,7$ and 8$)$. The data is shown in table (4) and depicted in Figure (12) with a notice that the transformer is considered operating in full load during the day hours and the wind convective heat transfer coefficient is taken as constant of a numerical value of $25 \mathrm{~W} / \mathrm{m}^{2}$. K [11]. It also should be noticed that the external heat which is obtained from equations 6,7 and 8 is considered as measured data because its base temperatures are measured directly on the finned body of transformer. The internal heat represents a theoretical data because their temperatures are obtained from the numerical scheme. 
Table (4) A comparison between internal and external heat transfer of transformer

\begin{tabular}{|c|c|c|c|c|c|}
\hline $\begin{array}{c}\text { Day } \\
\text { hours }\end{array}$ & $\begin{array}{c}\text { Oil Temp } \\
\left({ }^{\circ} \mathbf{C}\right) \\
\text { Equations } \\
\text { and 5) }\end{array}$ & $\begin{array}{c}\text { Body Temp } \\
(\mathbf{4} \mathbf{C}) \\
\text { Measured }\end{array}$ & $\begin{array}{c}\text { Internal heat } \\
(\mathbf{W}) \\
\text { Equations } \\
\text { and 5) }\end{array}$ & $\begin{array}{c}\text { External } \\
\text { heat }(\mathbf{W}) \\
\text { Equations 6, } \\
\mathbf{7} \text { and 8 }\end{array}$ & $\begin{array}{c}\text { ERROR } \\
\text { \% }\end{array}$ \\
\hline 6:00 AM & 52 & 55 & 2,890 & 2,740 & 4.28 \\
\hline 8:00 AM & 61 & 63 & 3,125 & 3,010 & 5.55 \\
\hline 10:00 AM & 68 & 70 & 3,670 & 3,480 & 2.63 \\
\hline 12:00 PM & 75 & 78 & 3,910 & 3,780 & 5.19 \\
\hline 2:00 PM & 82 & 85 & 4,230 & 4,185 & 1.19 \\
\hline 4:00 PM & 85 & 88 & 4,660 & 4,635 & 2.29 \\
\hline
\end{tabular}

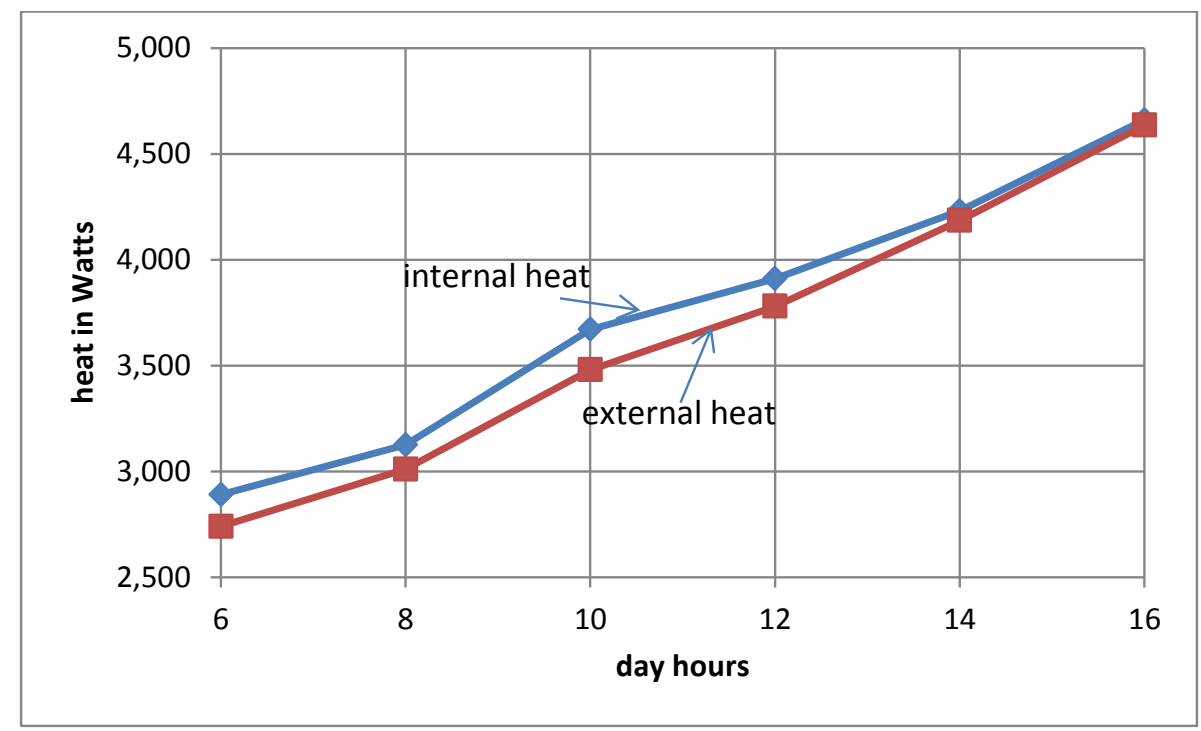

Fig. (12) a comparison between internal and external heats

The error associated with Figure (12) which can be calculated using equation 12 is shown in Figure (13). The data is almost identical at midday when the steady state is reached 


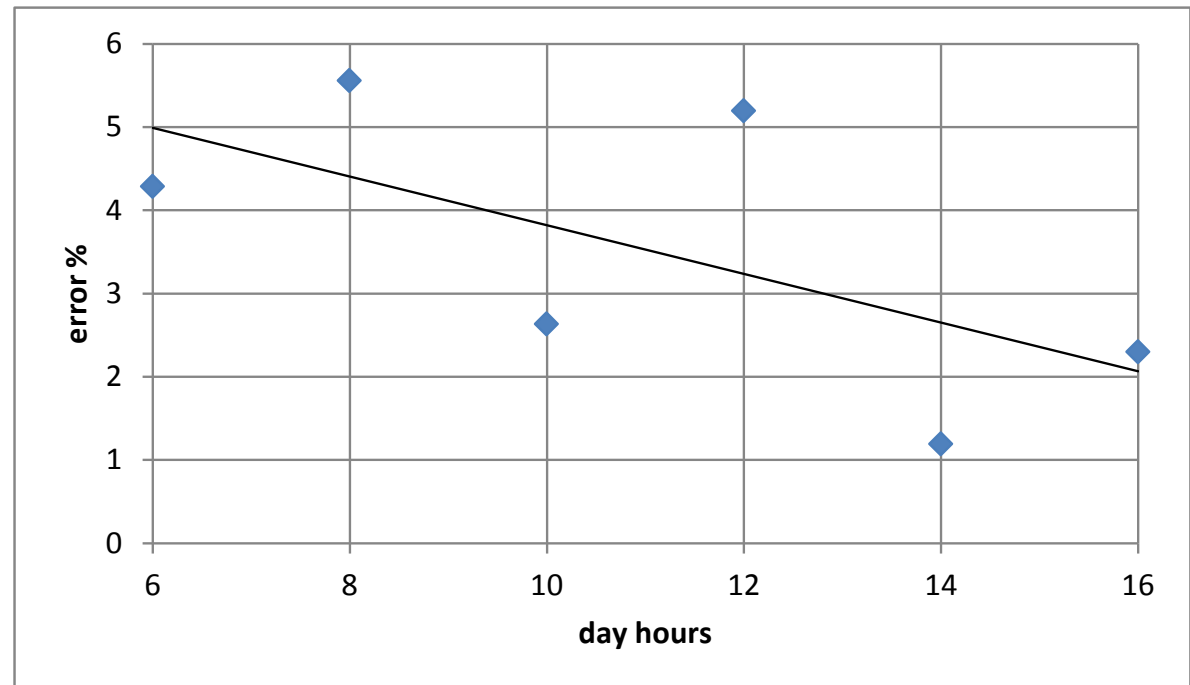

Figure (13) Percentage error associated with the data shown

The figures shown above reveal the fact that highest temperature inside a transformer occurs where the generating source of heat is located far from boundaries. This is valid for the phase which is located in the center of transformer tank (normally phase S). But this does not allow any modification because it will violate electromagnetic theory. Nevertheless, single phase transformers and V-Shaped iron core transformers (if there is any) where all phases located within the same distance from the boundaries will perform better heat transfer system than the conventional one.

Because both windings are installed co-axially, the peak temperature occurs within the half space which is occupied by LV windings. This was clearly shown in Figure (6) and is caused by the high value of current passing though LV windings compared to HV windings.

The vertical axis of transformer, which is normally installed outdoor, is exposed to open sun during day hours. Besides, hot oil always occupies the upper part of transformer tank. Hence, the peak temperature occurred in the upper part of transformer tank. This was clearly shown in Figure (5).

\section{Conclusion}

It can be now concluded that ambient temperature of $55^{\circ} \mathrm{C}$ which was listed in the specifications of Ministry of Electricity is no longer feasible and should be revised to $60^{\circ} \mathrm{C}$. This new ambient temperature will allow for wider range of oil temperature rise (see table 3) and accordingly higher temperature value for thermal trip and thermal wining resistance. The transformer under such high load and ambient temperature will be shut off by protection device (if it works properly). Otherwise, it will be out of service because of an internal damage in the windings most properly in Phase S depends on the load (if the thermal protection device does not work). 


\section{References}

[1] Pierce, L.W., "Hottest spot temperatures in ventilated dry type transformers", IEEE Transactions on Power Delivery, Vol. 9, No.1, January 1994.

[2] Nordman, H., "Average oil temperature rise in distribution transformers without external oil circulation”, Technical memorandum 2004.

[3] Susa D., Lethonen M., Nordman H., "Dynamic thermal modelling of power transformers", IEEE Transactions on Power Delivery, Vol. 20, No. 1, January 2005.

[4] El Wakil, N., Chereches, N.C., Padet, J.,"Numerical study of heat transfer and fluid flow in a power transformer", International Journal of Thermal Sciences, 45 pag. 615-626, 2006.

[5] Olsson O., C, Kranenborg, E.J., Samuelsson, B.R., "Numerical Study on Mixed Convection and Thermal Streaking in Power Transformer Windings", 5th European Thermal-Sciences Conference, The Netherlands, 2008.

[6] Haritha VV SS, T R Rao, Amit Jain, M Rammamorty, "Thermal Modeling of Electrical Utility", Third International Conference on Power Systems, Kharagpur, India, December 2009.

[7] Mahmmod A., "wavelet numerical scheme for solving one and two dimensional heat equation”, Ph.D. thesis, Gadjah Mada University, Indonesia, 2005.

[8] F. P. Incropera, D. P. DeWitt, "Fundamentals of heat and mass transfer", 4th edition, New York/USA: J. Wiley \& Sons, 1996.

[9] M. A. Taghikhani, A. Gholami, "Temperature Distribution in Power Transformer Windings with NDOF and DOF Cooling”, PES General Meeting”, Pittsburgh, PA, USA, 20-24 July, 2008.

[10] IEC Standard, IEC60076-7 (2006) "Loading guide for oil immersed transformers".

[11] J. P. Holman and Souvik Bhattacharyya, "Heat Transfer", Mc Graw Hill, India, 2011. 